\title{
Metastatic signet-ring cell cancer of the bladder responding to chemotherapy with capecitabine: case report and review of literature
}

\author{
Jorg Michels, MD, PhD; ${ }^{*}$ Sean Barbour, MD; ${ }^{\dagger}$ Douglas Cavers, MD; ${ }^{\ddagger}$ Kim N. Chi, MD
}

\begin{abstract}
Signet-ring cell cancers deriving from the bladder are rare entities and usually present with advanced incurable disease and associated poor outlook. No standard effective chemotherapeutic option has been described largely due to the rarity of this malignancy. We report a case of a patient with metastatic bladder cancer, signet-ring cell variant. The patient progressed rapidly on standard first-line bladder cancer chemotherapy with gemcitabine and carboplatin. He responded well to second-line capecitabine with a clinically meaningful progression-free survival.
\end{abstract}

Can Urol Assoc J 2010;4(2):E55-E57

\section{Introduction}

Bladder cancer is the fifth most common malignancy in Canada. ${ }^{1}$ Urothelial carcinoma of the transitional cell type is the most prevalent histological subtype in about $90 \%$ of patients, and the use of modern platinum-based combination chemotherapy has translated in significant clinical benefit in patients with advanced stage disease. ${ }^{2}$ Adenocarcinoma of the bladder comprises only $0.5 \%$ to $2 \%$ of all epithelial bladder tumours and primary signet-ring cell carcinoma, a variant of adenocarcinoma, is extremely rare with a prevalence estimated at $0.24 \%$ of primary bladder cancers; no effective systemic chemotherapy has been established for this subtype. ${ }^{3}$ We report a case of widespread metastatic signet-ring cell carcinoma variant of bladder cancer that responded well to second-line chemotherapy with capecitabine; we also review the literature.

\section{Case report}

An 81-year-old Asian man presented with obstructive urinary symptoms. Physical examination revealed an indurated prostate and inguinal lymphadenopathy. Computed tomography $(\mathrm{CT})$ demonstrated marked bladder wall thickening, an enlarged prostate, pelvic and inguinal lymphandenopa- thy, diffuse liver and bony metastases and a normal appearing gastrointestinal tract (Fig. 1, refer to $\mathrm{A} / \mathrm{C}$ ). A bone scan confirmed widespread metastases. Cystocopic evaluation of the bladder demonstrated no obvious mucosal lesion, but significant obstruction of the bladder neck. Pathology of transurethral resection specimen revealed prostatic tissue with diffuse infiltration by large areas of signet-ring carcinoma interspersed with poorly differentiated urothelial cancer (Fig. 2, refer to A/B).

Immunohistochemical staining of tumour tissue was negative for prostate-specific antigen (PSA) and prostatespecific acid phosphatase, strongly positive for cytokeratin (CK) $7 / 20$ / carcinoembryonic antigen (CEA), strong nuclear positive for caudal-related homeobox 2 (CDX2) and negative staining for CK5/6. Serum tumour marker panel revealed normal PSA and serum beta human chorionic gonadotropin (HCG) levels, elevated levels of CEA (12 $\mu \mathrm{g} / \mathrm{L}$, upper limit of normal $[\mathrm{ULN}]=4 \mu \mathrm{g} / \mathrm{L}$ ) and carbohydrate antigen (CA) 19-9 (160 kU/L, ULN = $36 \mathrm{kU} / \mathrm{L})$. Baseline renal function was impaired (calculated glomerular filtration rate of $42 \mathrm{~mL} / \mathrm{min}$ ) and liver function tests were normal.

The patient was started on combination chemotherapy of gemcitabine and carboplatin for TNM stage T4N2M1 poorly differentiated bladder cancer, signet-cell variant. He progressed after his first cycle of treatment with worsening of obstructive urinary symptoms, enlarging left inguinal lymph nodes, worsening of CEA and CA 19-9 levels (Fig. 3) and mild worsening of liver function tests (alkaline phosphates, aspartate aminotransferase and alanine transmaninase all less than 5 times the ULN). The patient received pelvic radiotherapy (2100 cGy in 3 fractions) for local control. He subsequently received 6 cycles of second-line chemotherapy with capecitabine. The patient tolerated treatment well, and his cancer-related symptoms normalized, as did his tumour markers and liver function tests. Post-treatment CT (Fig. 1, refer to $\mathrm{B} / \mathrm{D})$ confirmed response to treatment with significant shrinkage of the number and size of liver metastases, reduction in tumour-related bladder wall-thickening and lymphandenopathy. The patient was well post-chemotherapy and travelled to his family in China. Upon return, he developed 


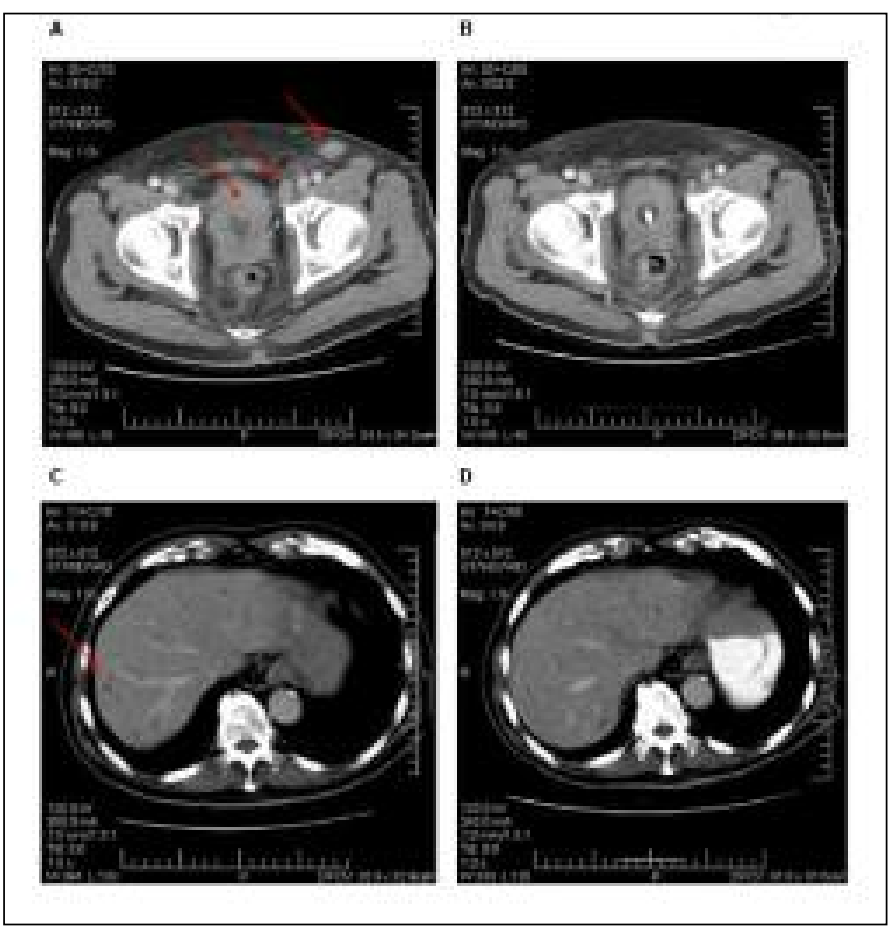

Fig. 1. Axial computer tomographic images. Corresponding imaging sections before $(A / C)$ and at end $(B / D)$ of capecitabine chemotherapy are demonstrated. Marked bladder wall thickening with significant pelvic/inguinal lymphadenopathy and multiple liver metastases are noted (red arrows) on baseline computer tomography.

progressive disease 9 months after he had started his first course of capecitabine. He was rechallenged with capecitabine and initially responded well, clinically and on tumour marker assessment (Fig. 3). Unfortunately, he deteriorated rapidly after his third cycle of treatment with tumourrelated obstructive jaundice and succumbed from liver failure 12 months after initial diagnosis.

\section{Discussion}

Pure primary signet-ring cell carcinomas of the bladder are very rare and only a few case reports exist of a mixed urothelial/signet-ring cell variant. ${ }^{3,4}$ Saphir first described primary signet-ring cell carcinoma of the bladder in 1955, and less than 200 cases have been reported in the literature since then. ${ }^{5,6}$ There is a male preponderance and most patients present at an advanced stage. This appears related to a linitis plastic-like growth pattern rendering cystoscopic detection difficult and the outlook poor. ${ }^{7}$

Adeno- or signet-ring cell carcinomas identified in the bladder are rare entities and may represent metastases from other primary sites, usually from the gastrointestinal tract. Clinical imaging and immunohistochemical findings in our patient were compatible with the primary source of signet cell cancer arising from the bladder. Our case demonstrated mixed histology with predominant signet-ring cells inter-

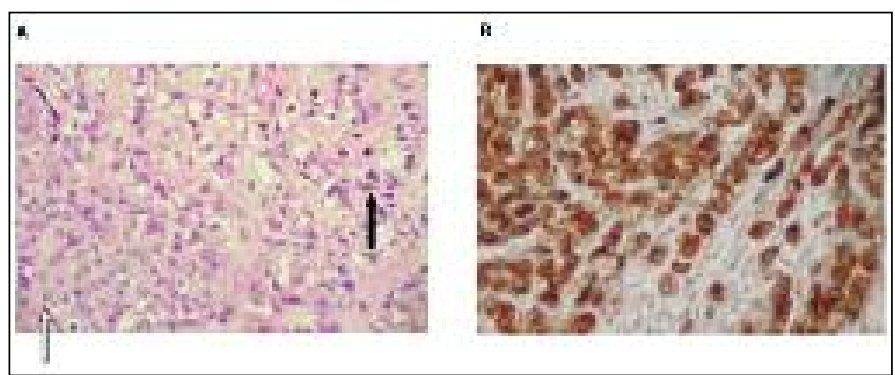

Fig. 2. Pathology and Immunohistochemistry. The microscopic presence of 2 different malignant cell populations $(A)$ is seen on hematoxylin and eosin stain on low power field (signet-ring cells are marked with open arrow, poorly differentiated urothelial carcinoma with closed arrow). Signet-ring cells stained positive for carcinoembryonic antigen on immunohistochemistry (B).

spersed with poorly differentiated urothelial cancer. Tumour tissue stained positive for $\mathrm{CK} 7 / 20$ and CDX2 but negative for PSA/Placental alkaline phosphatase (PLAP). Colorectal cancers are often CK7- but CK20+/CDX2+; prostate cancers are PSA+/PLAP+/CDX2-; and upper gastrointestinal tract tumours are usually CK $7+$ with variable CDX2/CK20 staining. ${ }^{8,9}$ The histogenesis of primary signet cell cancer in the bladder is not well-understood, and include metaplasia of transitional cell carcinoma. ${ }^{10,11}$ The coexistent signetring cell carcinoma and poorly differentiated urothelial cancer in our case may support such hypothesis.

Treatment of signet-ring variants of bladder cancer is not well-defined due to the rarity of the tumour. Cystectomy is indicated for localized disease and intra-arterial platinium or methotrexate-based chemotherapy with or without radiotherapy to control localized disease with prolonged progression-free survival have been reported. ${ }^{12,13}$ However, there is no systemic standard chemotherapy regimen for advanced disease, which in general is considered chemotherapy resistant. ${ }^{3}$ Our patient failed to respond to standard platinium-based chemotherapy for bladder cancer, but derived a meaningful clinical benefit from capecitabine, an orally bioavailable 5-fluorouracil (FU) prodrug. Case reports of signet-ring cell carcinoma originating from different primary sites but also adenocarcinomas of the bladder indicate similar experiences in as much that responses, if encountered, appear to follow 5-FU-based regimens. ${ }^{14-16}$

The kinetics of plasma CA19-9/CEA levels in our patient accurately matched the clinical and imaging behavior of the tumour. Elevated serum carbohydrate antigen 19-9 and CEA has been found to be elevated in signet-ring cell carcinoma in the past. ${ }^{17,18}$ Serum CA19-9 and CEA levels hence may provide simple and useful biomarkers to assess treatment efficacy in this tumour entity.

\section{Conclusion}

The overall outcome for signet-ring cell cancers in general is very poor. The significant response in our patient (follow- 


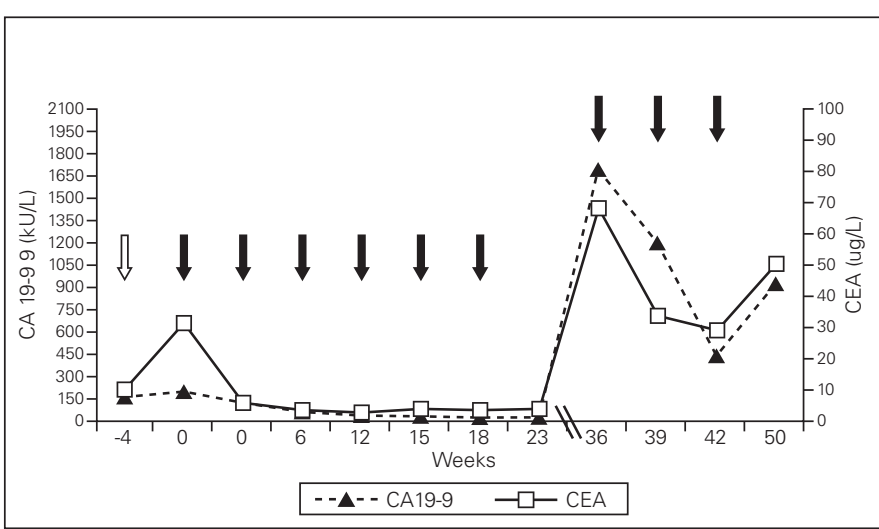

Fig. 3. Tumour marker kinetics during chemotherapy. Serial serum levels of carbohydrate antigen 19-9 and carcinoembryonic antigen are shown immediately prior to gemcitabine/carboplatin (open arrow) and following chemotherapy cycles with capecitabine (closed arrows).

ing capecitabine and after failing on standard chemotherapy for bladder cancer) and similar findings in the literature appear to indicate that 5-FU-based regimens should be considered the preferred first-line chemotherapeutic choice for patients with signet-ring cell transformation of bladder cancer.

*Medical Oncologist, Vancouver Island Centre, British Columbia Cancer Agency, Victoria, BC; †Internal Medicine resident, UBC Division of Nephrology, Vancouver, BC; P Pathologist (retired), Department of Laboratory Medicine and Pathology, Vancouver Island Health Authority, Victoria, BC; §Medical Oncologist, Vancouver Centre, British Columbia Cancer Agency, Vancouver, BC

Competing interests: None declared.

This paper has been peer-reviewed.

\section{References}

1. Marrett LD, De P, Airia P, et al.; for the Steering Committee of Canadian Cancer Statistics 2008. Cancer in Canada in 2008. CMAJ 2008;179:1163-70.

2. von der Maase H, Hansen SW, Roberts JT, et al. Gemcitabine and cisplatin versus methotrexate, vinblastine, doxorubicin, and cisplatin in advanced or metastatic bladder cancer: results of a large, randomized, multinational, multicenter, phase III study. J Clin Oncol 2000;18:3068-77.

3. Holmang S, Borghede G, Johansson SL. Primary signet-ring cell carcinoma of the bladder: a report on 10 cases. Scand J Urol Nephrol 1997;31:145-8.

4. Ozeki Z, Kobayashi S, Machida T, et al. Transitional cell carcinoma of the urinary bladder accompanied by signet-ring cell carcinoma: a case report [in Japanese]. Hinyokika Kiyo 2003;49:411-3.

5. Saphir 0. Signet-ring cell carcinoma of the urinary bladder. Am J Pathol 1955;31:223-31.

6. Fiter L, Gimeno F, Martin L, et al. Signet-ring cell adenocarcinoma of bladder. Urology 1993;41:30-3.

7. Torenbeek R, Koot RA, Blomious CE, et al. Primary signet-ring cell carcinoma of the urinary bladder. Histopathology 1996;28:33-40.

8. Jiang J, Ulbright TM, Younger C, et al. Cytokeratin 7 and cytokeratin 20 in primary urinary bladder carcinoma and matched lymph node metastasis. Arch Pathol Lab Med 2001;125:921-3.

9. Werling RW, Yaziii H, Bacchi CE, et al. CDX2, a highly sensitive and specific marker of adenocarcinomas of intestinal origin: an immunohistochemical survey of 476 primary and metastatic carcinomas. Am I Surg Pathol 2003;27:303-10.

10. Moll C, Landolt U, Pedio G. Signet-ring cell differentiation of transitional cell carcinomas of the bladder. Acta Cytol 1996;40:619-21.

11. Kunze E, Francksen B. Histogenesis of nonurothelial carcinomas of the urinary bladder from pre-existent transitional cell carcinomas. A histopathological and immunohistochemical study. Urol Res 2002;30:66-78.

12. Hirano Y, Suzuki K, Fujita K, et al. Primary signet-ring cell carcinoma of the urinary bladder successfully treated with intra-arterial chemotherapy alone. Urology 2002;59:601.

13. Krichen Makni $S$, Ellouz $S$, Khabir A, et al. Primary signet-ring cell carcinoma of urinary bladder. A case report [in French]. Cancer Radiother 2005;9:332-4.

14. Heidemann J, Gockel HR, Winde G, et al. Signet-ring cell carcinoma of unknown primary location. Metastatic to lower back musculature - remission following FU/FA chemotherapy. Z Gastroenterol 2002;40:33-6.

15. Mizoshita T, Kataoka H, Kubota E, et al. Gastric phenotype signet-ring cell carcinoma of the stomach with multiple bone metastases effectively treated with sequential methotrexate and 5-fluorouracil. Int J Clin Oncol 2008;13:373-6.

16. Logothetis $\mathrm{CJ}$, Samuels ML, Ogden S. Chemotherapy for adenocarcinomas of bladder and urachal origin: 5-Fluorouracil, doxorubicin, and mitomycin-C. Urology 1985;26:252-5.

17. el Sandid $M$, Peraldi $R$, Pernin $F$. Linitis plastica type of primary signet cell adenocarcinoma of the bladder [in French]. Prog Urol 2002;12:313-7.

18. Hiramatsu K, Mizukami Y, Momiyama M, et al. Stage IV gastric cancer patient who underwent palliative gastrectomy showing complete response to induction therapy with methotrexate plus 5 -fluorouracil and secondary treatment with oral TS-1 [in Japanese]. Gan To Kagaku Ryoho 2005;32:1163-6.

Correspondence: Dr. Jorg Michels, BC Cancer Agency, Vancouver Island Centre, 1410 Lee Ave., Victoria, BC V8R 6V5; fax: 250-519-2017; imichels@bccancer.bc.ca 